\title{
Robust STABILIZATION OF A QUADROTOR AERIAL VEHICLE IN PRESENCE OF SENSOR FAILURES
}

\author{
Hicham Khebbache ${ }^{1}$, Belkacem Sait $^{2}$ and Fouad Yacef ${ }^{3}$ \\ ${ }^{1,2}$ Automatic Laboratory of Setif (LAS), Electrical Engineering Department, \\ Setif University, ALGERIA \\ khebbachehicham@yahoo.fr, sait_belkacem19@yahoo.fr \\ ${ }^{3}$ Automatic Laboratory of Jijel (LAJ), Automatic Control Department, \\ Jijel University, ALGERIA \\ yaceffouadeyahoo.fr
}

\begin{abstract}
The work proposed in this paper aims to design a robust stabilization of an underactuated quadrotor UAV system in presence of sensor failures. The dynamical model of quadrotor while taking into account various physical phenomena, which can influence the dynamics of a flying structure is presented. Subsequently, a new control strategy based on backstepping approach is developed. Lyapunov based stability analysis shows that the proposed control strategy design keep the stability of the closed loop dynamics of the quadrotor UAV even after the presence of sensor failures. Numerical simulation results are provided to show the good tracking performance of proposed control laws.
\end{abstract}

\section{KEYWORDS}

Backstepping, Dynamic model of quadrotor, Fault tolerant control (FTC), Robust control, Sliding mode, Sensor failures, Unmanned aerial vehicles $(U A V)$.

\section{INTRODUCTION}

The automatic control of a quadrotor UAV is not a straight on mainly due to its underactuated properties. The dynamic model of quadrotor UAV has six degree-of-freedom (DOF) with only four independent thrust forces generated by four rotors. It is difficult to control all these six outputs with only four control inputs. Moreover, uncertainties associate with dynamic model also bring more challenge for control design.

Despite the real progress made, researchers must still deal with serious difficulties, related to the control of such systems, particularly, in the presence of atmospheric turbulences. In addition, the navigation problem is complex and requires the perception of an often constrained and evolutionary environment, especially in the case of low-altitude flights.

The quadrotors, has been studied recently by some authors [14], [8], [2], [15], [16], [11], [20], [3], [4], [1], [6], [12], [17], [10], [13], [9], [18], [7], [5]. These systems as many other dynamic systems, present constant or slowly-varying uncertain parameters, but these authors do not take into account the failures affecting the sensors of our system, wich makes them very limited and induces undesired behavior of quadrotor, or even to instability of the latter after occurence of sensor faults.

In this paper, the stabilization problem of the quadrotor aircraft in presence of sensor failures is considered. The dynamical model describing the quadrotor aircraft motions and taking into a

DOI : 10.5121/ijctcm.2012.2204 
account for various parameters which affect the dynamics of a flying structure is presented. Subsequently, a new control strategy based on backstepping approach taking into account the sensor failures is developed. In order to compensate the effects of these failures, this control strategy includes two compensation techniques, the first one using an integral term, the second, is to use an another term containing "sign" function. Finally all synthesized control laws are highlighted by simulations which gave fairly satisfactory results despite the occurrence of sensor failures.

\section{DYNAMiCAL MODEL}

\subsection{Quadrotor dynamic model}

The quadrotor have four propellers in cross configuration. The two pairs of propellers $\{1,3\}$ and $\{2,4\}$ as described in Figure. 1, turn in opposite directions. By varying the rotor speed, one can change the lift force and create motion. Thus, increasing or decreasing the four propeller's speeds together generates vertical motion. Changing the 2 and 4 propeller's speed conversely produces roll rotation coupled with lateral motion. Pitch rotation and the corresponding lateral motion; result from 1 and 3 propeller's speed conversely modified. Yaw rotation is more subtle, as it results from the difference in the counter-torque between each pair of propellers.

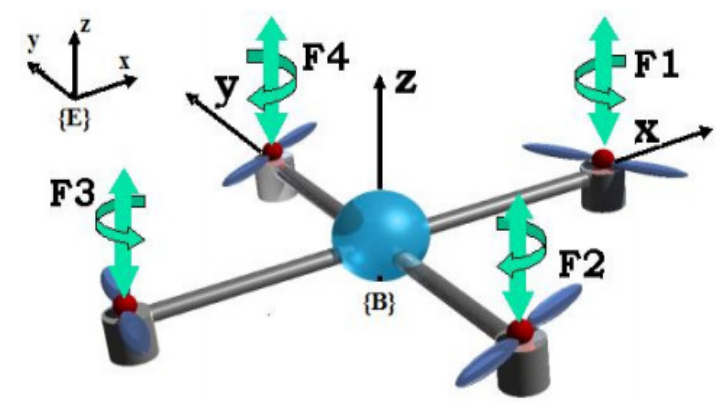

Figure 1. Quadrotor configuration

The quadrotor model (position and orientation dynamic) obtained is given like in [3], [4], [5] by:

$$
\left\{\begin{array}{l}
\ddot{\phi}=\frac{\left(I_{y}-I_{z}\right)}{I_{x}} \dot{\theta} \dot{\psi}-\frac{J_{r}}{I_{x}} \bar{\Omega}_{r} \dot{\theta}-\frac{K_{f a x}}{I_{x}} \dot{\phi}^{2}+\frac{l}{I_{x}} u_{2} \\
\ddot{\theta}=\frac{\left(I_{z}-I_{x}\right)}{I_{y}} \dot{\phi} \dot{\psi}+\frac{J_{r}}{I_{y}} \bar{\Omega}_{r} \dot{\phi}-\frac{K_{f a y}}{I_{y}} \dot{\theta}^{2}+\frac{l}{I_{y}} u_{3} \\
\ddot{\psi}=\frac{\left(I_{x}-I_{y}\right)}{I_{z}} \dot{\theta} \dot{\phi}-\frac{K_{f a z}}{I_{z}} \dot{\psi}^{2}+\frac{1}{I_{z}} u_{4} \\
\ddot{x}=-\frac{K_{f t x}}{m} \dot{x}+\frac{1}{m} u_{x} u_{1} \\
\ddot{y}=-\frac{K_{f t y}}{m} \dot{y}+\frac{1}{m} u_{y} u_{1} \\
\ddot{z}=-\frac{K_{f t z}}{m} \dot{z}-g+\frac{\cos (\phi) \cos (\theta)}{m} u_{1}
\end{array}\right.
$$

with 


$$
\left\{\begin{array}{l}
\bar{\Omega}_{r}=\omega_{1}-\omega_{2}+\omega_{3}-\omega_{4} \\
u_{x}=(\cos \phi \cos \psi \sin \theta+\sin \phi \sin \psi) \\
u_{y}=(\cos \phi \sin \theta \sin \psi-\sin \phi \cos \psi)
\end{array}\right.
$$

$u_{1}, u_{2}, u_{3}$ and $u_{4}$ are the control inputs of the system which are written according to the angular velocities of the four rotors as follows:

$$
\left[\begin{array}{l}
u_{1} \\
u_{2} \\
u_{3} \\
u_{4}
\end{array}\right]=\left[\begin{array}{cccc}
b & b & b & b \\
0 & -l b & 0 & l b \\
-l b & 0 & l b & 0 \\
d & -d & d & -d
\end{array}\right]\left[\begin{array}{l}
\omega_{1}^{2} \\
\omega_{2}^{2} \\
\omega_{3}^{2} \\
\omega_{4}^{2}
\end{array}\right]
$$

From (2) it easy to show that :

$$
\left\{\begin{array}{l}
\phi_{d}=\arcsin \left(u_{x} \sin \left(\psi_{d}\right)-u_{y} \cos \left(\psi_{d}\right)\right) \\
\theta_{d}=\arcsin \left(\frac{\left(u_{x} \cos \left(\psi_{d}\right)+u_{y} \sin \left(\psi_{d}\right)\right)}{\cos \left(\phi_{d}\right)}\right)
\end{array}\right.
$$

\subsection{Rotors dynamic model}

The dynamics of a DC motor is given by the following differential equations:

$$
J_{r} \dot{\omega}_{i}=\tau_{i}-Q_{i}, i \in\{1,2,3,4\}
$$

With $Q_{i}=d \omega_{i}^{2}$ is the reactive torque generated, in free air, by the rotor $i$ due to rotor drag, and $\tau_{i}$ is the input torque.

A control law for the input torque $\tau_{i}$ is developed in [20], it is given by:

$$
\tau_{i}=Q_{i}+J_{r} \dot{\omega}_{d, i}-k_{i} \tilde{\omega}_{i}
$$

where $k_{i}, \mathrm{i} \in\{1, \ldots, 4\}$ are four positive parameters, $\omega_{d, i}, \mathrm{i} \in\{1, \ldots, 4\}$ are the desired speed of each rotor and $\tilde{\omega}_{i}=\omega_{i}-\omega_{d, i}$.

In fact, applying (6) to (5) leads to

$$
\dot{\tilde{\omega}}_{i}=-\frac{k_{i}}{J_{r}} \tilde{\omega}_{i}
$$

which shows the exponential convergence of $\omega_{i}$ to $\omega_{d, i}$ and hence the convergence of the airframe torques to the desired values leading to the attitude stabilization of the quadrotor aircraft.

In our application, the DC motors are voltage controlled. Assuming that the motor inductance is small and taking into consideration the gear ratio, one can obtain the voltage to be applied to each motor as follows [20]:

$$
v_{i}=\frac{R_{a}}{k_{m} k_{g}} \tau_{i}+k_{m} k_{g} \omega_{i}, i \in\{1,2,3,4\}
$$

where $R_{a}$ is the motor resistance, $k_{m}$ is the motor torque constant, and $k_{g}$ is the gear ratio.

\section{CONTROL STRATEGY OF QUADROTOR WITH ACTUATOR FAULTS}

The complete model resulting by adding the sensor faults in the model (1) can be written in the state-space form: 
International Journal of Control Theory and Computer Modelling (IJCTCM) Vol.2, No.2, March 2012

$$
\left\{\begin{array}{l}
\dot{x}(t)=\eta(x, t)+g(x, t) u(t) \\
y(t)=h(x, t)+f_{s}(t)
\end{array}\right.
$$

With $x(t) \in \mathfrak{R}^{n}$ is the state vector of the system, $y(t) \in \mathfrak{R}^{p}$ is the measured output vector, $u(t) \in \mathfrak{R}^{m}$ is the input control vector, and $f_{s}(t) \in \mathfrak{R}^{q}$ is the sensor failures vector, such as:

$$
x=\left[x_{1}, \ldots, x_{12}\right]^{T}=[\phi, \dot{\phi}, \theta, \dot{\theta}, \psi, \dot{\psi}, x, \dot{x}, y, \dot{y}, z, \dot{z}]^{T}
$$

Remark 1: In our contribution, only the velocity sensor failures are considered.

From (1), (10) and considering the velocity sensor faults, we obtain :

$$
\begin{aligned}
& \left\{\begin{array}{l}
\dot{x}_{1}=x_{2} \\
\dot{x}_{2}=a_{1} x_{4} x_{6}+a_{2} x_{2}^{2}+a_{3} \bar{\Omega}_{r} x_{4}+b_{1} u_{2} \\
\dot{x}_{3}=x_{4} \\
\dot{x}_{4}=a_{4} x_{2} x_{6}+a_{5} x_{4}^{2}+a_{6} \bar{\Omega}_{r} x_{2}+b_{2} u_{3} \\
\dot{x}_{5}=x_{6} \\
\dot{x}_{6}=a_{7} x_{2} x_{4}+a_{8} x_{6}^{2}+b_{3} u_{4} \\
\dot{x}_{7}=x_{8} \\
\dot{x}_{8}=a_{9} x_{8}+\frac{1}{m} u_{x} u_{1} \\
\dot{x}_{9}=x_{10} \\
\dot{x}_{10}=a_{10} x_{10}+\frac{1}{m} u_{y} u_{1} \\
\dot{x}_{11}=x_{12} \\
\dot{x}_{12}=a_{11} x_{12}-g+\frac{\cos (\phi) \cos (\theta)}{m} u_{1} \\
y=\left[\begin{array}{lllll}
x_{1} & x_{2}+f_{s 1} \quad x_{3} \quad x_{4}+f_{s 2} & x_{5} & x_{6}+f_{s 3}
\end{array}\right. \\
x_{7} \quad x_{8}+f_{s 4} \quad x_{9} \quad x_{10}+f_{s 5} \quad x_{11} \quad x_{12}+f_{s 6}
\end{array}\right]^{T}
\end{aligned}
$$

with

$$
\left\{\begin{array}{l}
a_{1}=\left(\frac{I_{y}-I_{z}}{I_{x}}\right) a_{2}=-\frac{K_{f a x}}{I_{x}} a_{3}=-\frac{J_{r}}{I_{x}} a_{4}=\left(\frac{I_{z}-I_{x}}{I_{y}}\right) a_{5}=-\frac{K_{f a y}}{I_{y}} \\
a_{6}=\frac{J_{r}}{I_{y}} a_{7}=\left(\frac{I_{x}-I_{y}}{I_{z}}\right) a_{8}=-\frac{K_{f a z}}{I_{z}} a_{9}=-\frac{K_{f f x}}{m} a_{10}=-\frac{K_{f t y}}{m} \\
a_{11}=-\frac{K_{f t z}}{m} b_{1}=\frac{l}{I_{x}} b_{2}=\frac{l}{I_{y}} b_{3}=\frac{1}{I_{z}}
\end{array}\right.
$$

The following assumptions is needed for the analysis,

Assumption 1: The velocity sensor failures are bounded,

$$
\left|f_{s i}(t)\right| \leq f_{s i}^{+}, \quad i \in[1,2,3,4,5,6]
$$

where $\left\{f_{s 1}{ }^{+},{f_{s 2}}^{+},{f_{s 3}}^{+}, f_{s 4}{ }^{+}, f_{s 5}{ }^{+}, f_{s 6}{ }^{+}\right\}$are positive constants. 
Assumption 2: The velocity sensor failures are slowly varying in time, as follows:

$$
\dot{f_{s i}}(t) \approx 0, \quad \mathrm{i} \in[1,2,3,4,5,6]
$$

The adopted control strategy is based on two loops (internal loop and external loop). The internal loop contains four control laws: control of roll, control of pitch, control of yaw and control of altitude. The external loop includes two control laws of positions $x$ and $y$. The external control loop generates a desired of roll $\left(\phi_{d}\right)$ and pitch $\left(\theta_{d}\right)$ through the correction block (illustrated by equation (4)). This block corrects the rotation of the roll and pitch depending on the desired yaw $\left(\psi_{d}\right)$. The synoptic scheme below shows this control strategy:

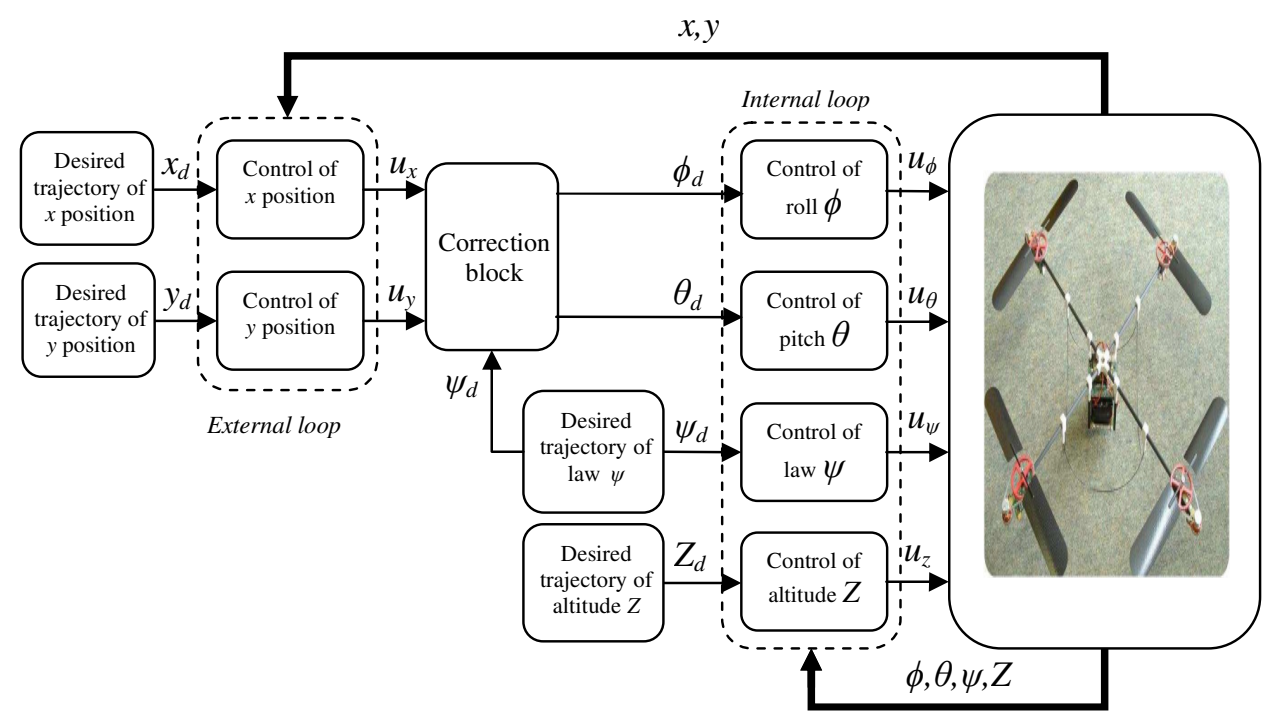

Figure 2. Synoptic scheme of the proposed control strategy

Basing on backstepping approach, a recursive algorithm is used to synthesize the control laws forcing the system to follow the desired trajectory in presence of velocity sensor failures, we simplify all stages of calculation concerning the tracking errors and Lyapunov functions in the following way:

$$
e_{i}= \begin{cases}x_{i}-x_{i d} & \mathrm{i} \in[1,3,5,7,9,11] \\ y_{i}-\dot{x}_{(i-1) d}+c_{(i-1)} e_{(i-1)}-\varsigma_{(i-1)} & \mathrm{i} \in[2,4,6,8,10,12]\end{cases}
$$

and

$$
\varsigma_{i}= \begin{cases}k_{s i} \int_{0}^{t} e_{i} d \tau & \mathrm{i} \in[1,3,5,7,9,11] \\ k_{s i} \operatorname{sign}\left(e_{i}\right) & \mathrm{i} \in[2,4,6,8,10,12]\end{cases}
$$

The corresponding lyapunov functions are given by:

$$
V_{i}= \begin{cases}\frac{1}{2} e_{i}^{2}+\frac{1}{2} e_{f j}^{2} & \mathrm{i} \in[1,3,5,7,9,11] \text { and } \mathrm{j} \in[1, \ldots, 6] \\ V_{i-1}+\frac{1}{2} e_{i}^{2} & \mathrm{i} \in[2,4,6,8,10,12]\end{cases}
$$


International Journal of Control Theory and Computer Modelling (IJCTCM) Vol.2, No.2, March 2012 such as

$$
\begin{cases}e_{f j}=f_{s j}-\varsigma_{i} & \mathrm{i} \in[1,3,5,7,9,11] \text { and } \mathrm{j} \in[1, \ldots, 6] \\
\Upsilon_{j}=\left(\begin{array}{cc}
c_{i} & k_{s i} \\
1 & 0
\end{array}\right)>0 & \mathrm{i} \in[1,3,5,7,9,11] \text { and } \mathrm{j} \in[1, \ldots, 6] \\
c_{i}>0 \text { and } k_{s i}>0 & \mathrm{i} \in[2,4,6,8,10,12]\end{cases}
$$

To synthesize a stabilizing control laws in the presence of velocity sensor failures, the following necessary condition must be verified:

$$
\left|\gamma_{s j}\right|<k_{s i}, \quad \mathrm{i} \in[2,4,6,8,10,12] \text { and } \mathrm{j} \in[1, \ldots, 6]
$$

$\left(\gamma_{s j}\right)$ represent the unknowns parts related to velocity sensor failures.

The synthesized stabilizing control laws are as follows:

$$
\left\{\begin{array}{l}
u_{2}=\frac{1}{b_{1}}\left(\ddot{x}_{1 d}-c_{1}\left(-c_{1} e_{1}+k_{s 1} \int_{0}^{t} e_{1} d \tau+e_{2}\right)+\left(k_{s 1}-1\right) e_{1}-c_{2} e_{2}-a_{1} y_{4} y_{6}-a_{2} y_{2}^{2}-a_{3} \bar{\Omega}_{r} y_{4}-k_{s 2} \operatorname{sign}\left(e_{2}\right)\right) \\
u_{3}=\frac{1}{b_{2}}\left(\ddot{\theta}_{d}-c_{3}\left(-c_{3} e_{3}+k_{s 3} \int_{0}^{t} e_{3} d \tau+e_{4}\right)+\left(k_{s 3}-1\right) e_{3}-c_{4} e_{4}-a_{4} y_{2} y_{6}-a_{5} y_{4}^{2}-a_{6} \bar{\Omega}_{r} y_{2}-k_{s 4} \operatorname{sign}\left(e_{4}\right)\right) \\
u_{4}=\frac{1}{b_{3}}\left(\ddot{\psi}_{d}-c_{5}\left(-c_{5} e_{5}+k_{s 5} \int_{0}^{t} e_{5} d \tau+e_{6}\right)+\left(k_{s 5}-1\right) e_{5}-c_{6} e_{6}-a_{7} y_{2} y_{4}-a_{8} y_{6}^{2}-k_{s 6} \operatorname{sign}\left(e_{6}\right)\right) \\
u_{x}=\frac{m}{u_{1}}\left(\ddot{x}_{d}-c_{7}\left(-c_{7} e_{7}+k_{s 7} \int_{0}^{t} e_{7} d \tau+e_{8}\right)+\left(k_{s 7}-1\right) e_{7}-c_{8} e_{8}-a_{9} y_{8}-k_{s 8} \operatorname{sign}\left(e_{8}\right)\right) \\
u_{y}=\frac{m}{u_{1}}\left(\ddot{y}_{d}-c_{9}\left(-c_{9} e_{9}+k_{s 9} \int_{0}^{t} e_{9} d \tau+e_{10}\right)+\left(k_{s 9}-1\right) e_{9}-c_{10} e_{10}-a_{10} y_{10}-k_{s 10} \operatorname{sign}\left(e_{10}\right)\right) \\
u_{1}=\frac{1 u_{1} \neq 0}{\cos \left(x_{1}\right) \cos \left(x_{3}\right)}\left(\ddot{z}_{d}-c_{11}\left(-c_{11} e_{11}+k_{s 11} \int_{0}^{t} e_{11} d \tau+e_{12}\right)+\left(k_{s 11}-1\right) e_{11}-c_{12} e_{12}-a_{11} y_{12}+g-k_{s 12} \operatorname{sign}\left(e_{12}\right)\right)
\end{array}\right.
$$

\section{- Proof}

For $i=1:$

$$
\left\{\begin{array}{l}
e_{1}=x_{1}-x_{1 d} \\
V_{1}=\frac{1}{2} e_{1}^{2}+\frac{1}{2} e_{f 1}^{2} / e_{f 1}=f_{s 1}-\varsigma_{1}
\end{array}\right.
$$

and

$$
\dot{V_{1}}=e_{1} \dot{e}_{1}+e_{f 1} \dot{e}_{f 1}=e_{1}\left(\left(y_{2}-f_{s 1}\right)-\dot{x}_{1 d}\right)+e_{f 1}\left(-\dot{\zeta}_{1}\right)
$$

The stabilization of $e_{1}$ can be obtained by introducing a new virtual control $y_{2}$

Consequently,

$$
\left(y_{2}\right)_{d}=\alpha_{1}=\dot{x}_{1 d}-c_{1} e_{1}+\varsigma_{1}
$$

$$
\dot{V_{1}}=e_{1}\left(-c_{1} e_{1}-e_{f 1}\right)+e_{f 1}\left(-\dot{\zeta}_{1}\right)
$$

In order to compensate the effect of the velocity sensor fault of roll motion, an integral term is introduced which can eliminate the tracking error. We take: 
International Journal of Control Theory and Computer Modelling (IJCTCM) Vol.2, No.2, March 2012

$$
\varsigma_{1}=k_{s 1} \int_{0}^{t} e_{1} d \tau
$$

It results that:

$$
\dot{V_{1}}=e_{1}\left(-c_{1} e_{1}-e_{f 1}\right)+e_{f 1}\left(-k_{s 1} e_{1}\right)=-\left(\begin{array}{ll}
e_{1} & e_{f 1}
\end{array}\right)\left(\begin{array}{cc}
c_{1} & 1 \\
k_{s 1} & 0
\end{array}\right)\left(\begin{array}{c}
e_{1} \\
e_{f 1}
\end{array}\right)=-\bar{e}_{1}^{T} \Upsilon_{1} \overline{e_{1}}
$$

$c_{1}$ and $k_{s 1}$ are chosen so as to make the matrix $\Upsilon_{1}$ positive definite, which means that, $V_{1} \leq 0$

For $i=2$ :

and

$$
\left\{\begin{array}{l}
e_{2}=y_{2}-\dot{x}_{1 d}+c_{1} e_{1}-\varsigma_{1} \\
V_{2}=V_{1}+\frac{1}{2} e_{2}^{2}
\end{array}\right.
$$

$$
\begin{aligned}
\dot{V}_{2} & =e_{1}\left(-c_{1} e_{1}-e_{f 1}+e_{2}\right)+e_{f 1}\left(-k_{s 1} e_{1}\right) \\
& +e_{2}\left(a_{1} y_{4} y_{6}+a_{2} y_{2}^{2}+a_{3} \bar{\Omega}_{r} y_{4}-\ddot{x}_{1 d}+c_{1}\left(-c_{1} e_{1}+\varsigma_{1}+e_{2}\right)-k_{s 1} e_{1}+\gamma_{s 1}+b_{1} u_{2}\right)
\end{aligned}
$$

We know a priori from (19) that:

$$
\left|\gamma_{s 1}\right|=\left|-c_{1} f_{s 1}-a_{1}\left(f_{s 3} y_{4}+f_{s 2} y_{6}-f_{s 2} f_{s 3}\right)-a_{2}\left(f_{s 1} y_{2}-f_{s 1}^{2}\right)-a_{3} \bar{\Omega}_{r} f_{s 2}\right|<k_{s 2}
$$

The stabilization of $\left(e_{1}, e_{2}\right)$ can be obtained by introducing the input control $u_{2}$

$$
u_{2}=\frac{1}{b_{1}}\left(\ddot{x}_{1 d}-c_{1}\left(-c_{1} e_{1}+\varsigma_{1}+e_{2}\right)+\left(k_{s 1}-1\right) e_{1}-c_{2} e_{2}-a_{1} y_{4} y_{6}-a_{2} y_{2}^{2}-a_{3} \bar{\Omega}_{r} y_{4}-\varsigma_{2}\right) / c_{2}>0
$$

It result that

$$
\dot{V}_{2}=-\bar{e}_{1}^{T} \Upsilon_{1} \bar{e}_{1}-c_{2} e_{2}^{2}-e_{2}\left(\varsigma_{1}-\gamma_{s 1}\right)
$$

In order to compensate the unknown part related to velocity sensor faults $\left(\gamma_{s 1}\right)$, a "sign" function is introduced. We take:

$$
\varsigma_{2}=k_{s 2} \operatorname{sign}\left(e_{2}\right)
$$

By using the compensation term (32) and the equation (31) it comes

$$
\dot{V}_{2} \leq-\bar{e}_{1}^{T} \Upsilon_{1} \bar{e}_{1}-c_{2} e_{2}^{2}-\left|e_{2}\right|\left(k_{s 2}-\left|\gamma_{s 1}\right|\right) \leq 0
$$

The same steps are followed to extract $u_{3}, u_{4}, u_{x}, u_{y}$ and $u_{1}$.

It is well known that sliding mode control signal is discontinuous in nature on the switching manifold, which in duces a chattering phenomenon. To avoid the effect of this phenomenon, the sign function can be replaced by another function called "saturation function sat(.)", it's is given by:

$$
\operatorname{sat}(e)= \begin{cases}\frac{e}{\Phi} & \text { if }|e| \leq \Phi \\ \operatorname{sign}(e) & \text { else }\end{cases}
$$




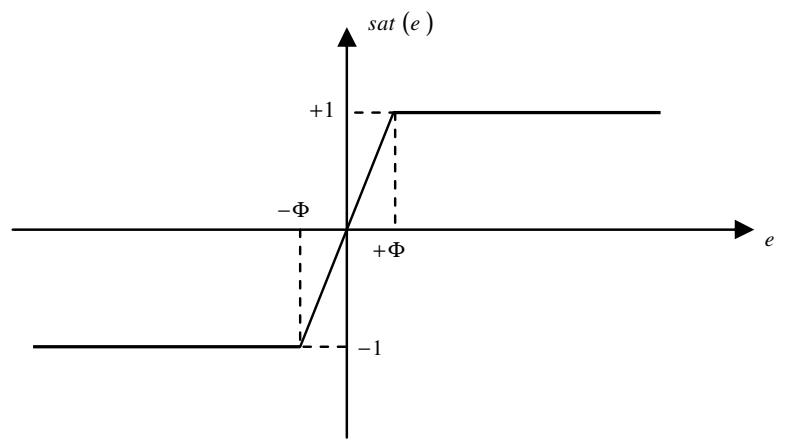

Figure. 3. Saturation function "sat(.)".

\section{SimUlation RESULTS}

In order to see the performances of the controller developed in this paper, two Tests are treated.

1- Results without faults are shown in Figure. 4, Figure. 5, Figure. 6 and Figure .10.a.

2- Results with four sensor faults $\left\{f_{s 1}, f_{s 2}, f_{s 3}, f_{s 6}\right\}$ added in angular velocities and linear velocity of altitude of with $50 \%$ of these maximum values at $20 \mathrm{~s}, 25 \mathrm{~s}, 30 \mathrm{~s}$ and $35 \mathrm{~s}$ respectively are shown in Figure. 7, Figure. 8, Figure. 9 and Figure.10.b.

The simulation results are obtained based on the real parameters in Table. 1 (see the appendix).

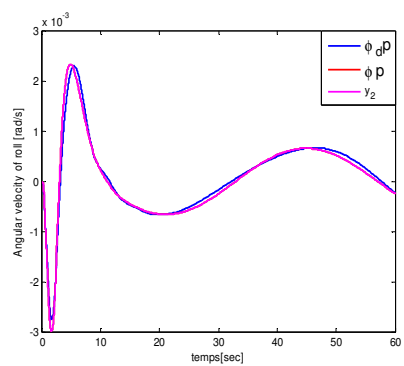

(a) evolution of angular velocity of roll

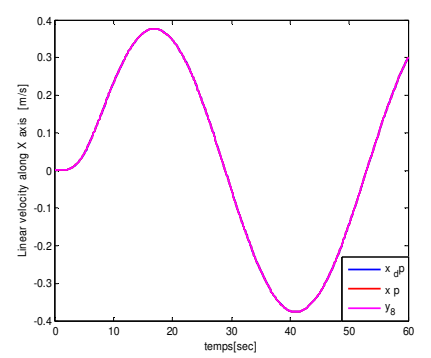

(d) evolution of linear velocity along $X$ axis

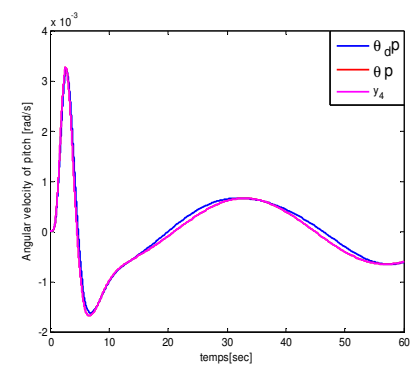

(b) evolution of angular velocity of pitch

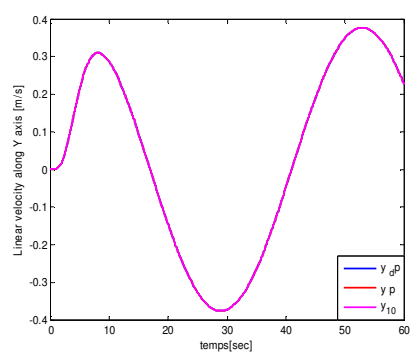

(e) evolution of linear velocity along $Y$ axis

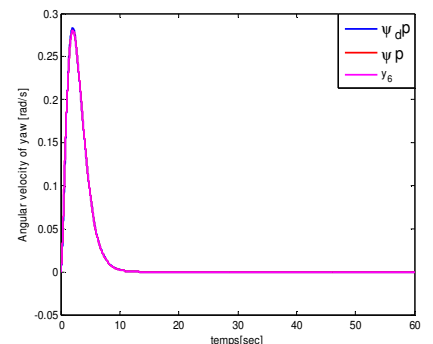

(c) evolution of angular velocity of yaw

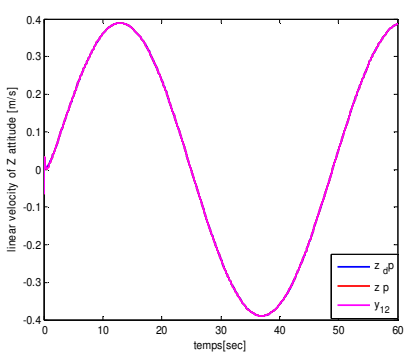

(f) evolution of linear velocity along $Z$ axis

Figure. 4. Tracking simulation results of the angular and linear velocities, Test 1.

Figure. 4 and Figure. 7 represents the quadrotor velocities, It can be seen a good tracking of the desired velocities in Figure. 4, with tracking deviation in the measurements of angular velocities and linear velocity of altitude with 50\% of her maximum values in Figure. 7 (illustrated respectively by (a), (b), (c), and (f)) after occurrence of these corresponding sensor failures, we can see also from this figure the small transient peaks in roll and pitch velocities due after 
occurrence of velocity sensor fault of altitude at $35 \mathrm{~s}$, which gives us a wrong information of velocities of our system.

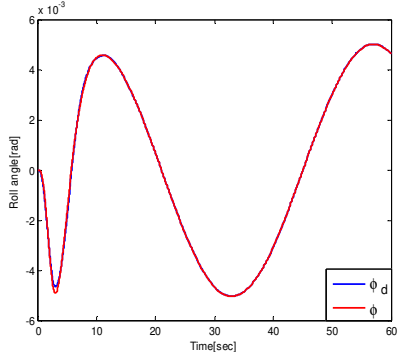

(a) evolution of roll angle

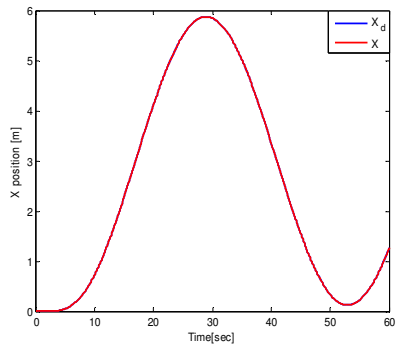

(d) evolution of $x$ position

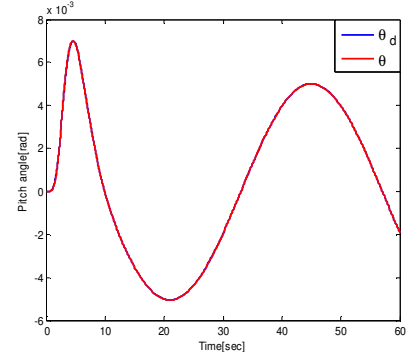

(b) evolution of pitch angle

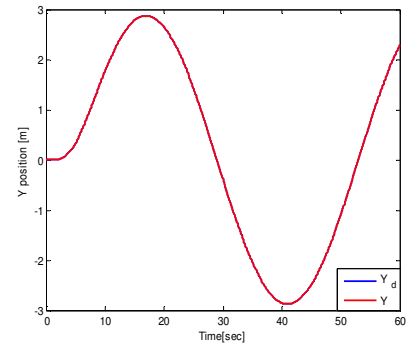

(e) evolution of $y$ position

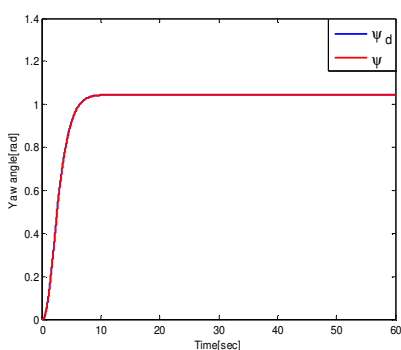

(c) evolution of yaw angle

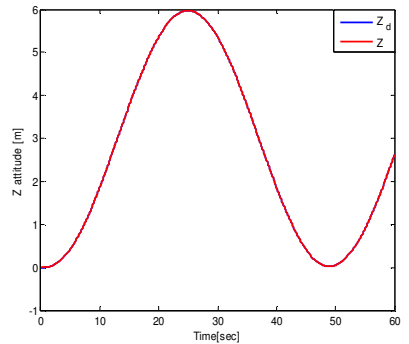

(f) evolution of $z$ position

Figure. 5. Tracking simulation results of trajectories along roll $(\phi)$, pitch $(\theta)$, yaw angle $(\psi)$ and $(\mathrm{X}, \mathrm{Y}, \mathrm{Z})$ axis, Test 1.

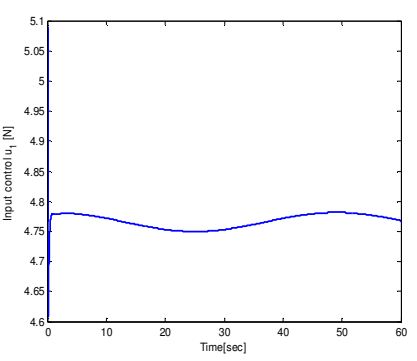

(a) evolution of input control $u_{1}$

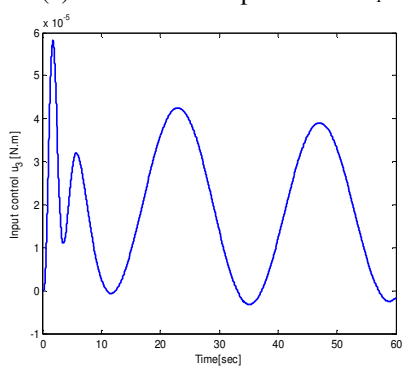

(c) evolution of input control $u_{3}$

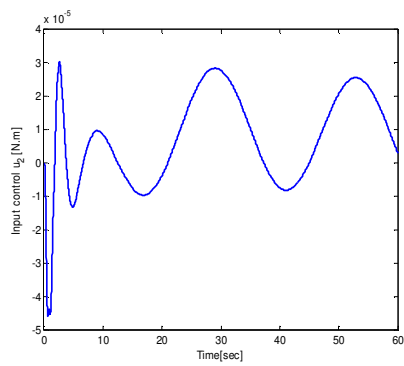

(b) evolution of input control $u_{2}$

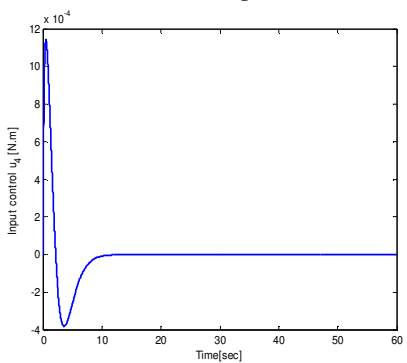

(d) evolution of input control $u_{4}$

Figure. 6. Simulation results of all controllers, Test 1.

Figure. 5 and Figure. 8 represents the quadrotor trajectories. From these figures, we can see well a good tracking of the desired trajectories, with small transient deviations in roll, pitch, yaw and altitude motions given by Figure. 8 (illustrated respectively by (a), (b), (c), and (f)) caused by the appearance of velocity sensor failures in angular velocities and linear velocity of altitude (illustrated respectively by (a), (b), (c), and (f) from Figure. 7) at 20sec, $25 \mathrm{sec}, 30 \mathrm{sec}$ and $35 \mathrm{sec}$ 
International Journal of Control Theory and Computer Modelling (IJCTCM) Vol.2, No.2, March 2012

respectively, these deviations converge quickly to their desired values, which also explains the robustness of this control approach toward the velocity sensor failures.

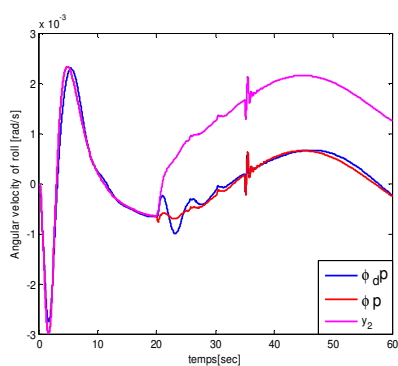

(a) evolution of angular velocity of roll

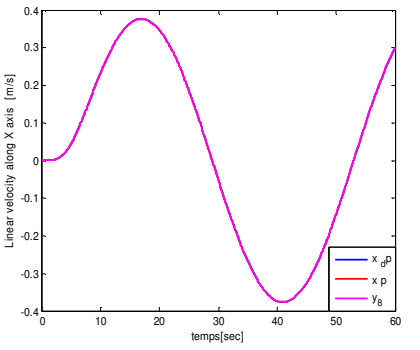

(d) evolution of linear velocity along $X$ axis

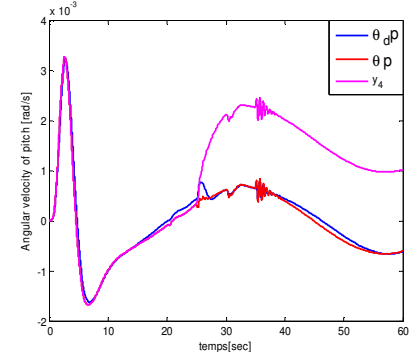

(b) evolution of angular velocity of pitch

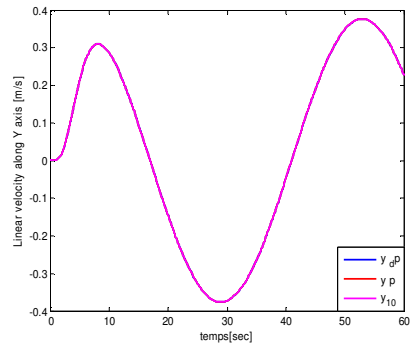

(e) evolution of linear velocity along $Y$ axis

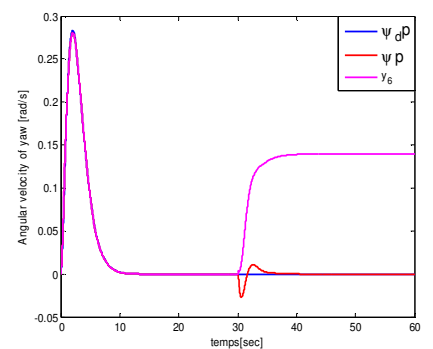

(c) evolution of angular velocity of yaw

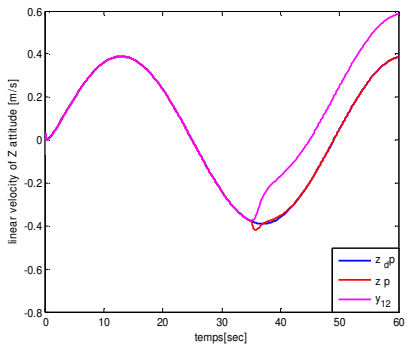

(f) evolution of linear velocity along $Z$ axis

Figure. 7. Tracking simulation results of the angular and linear velocities, Test 2.

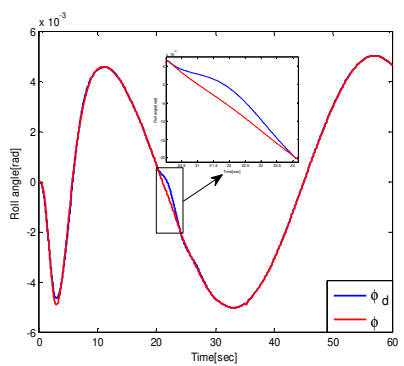

(a) evolution of roll angle

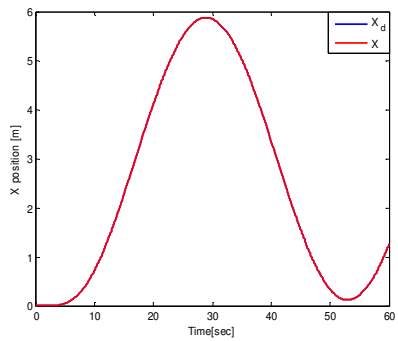

(d) evolution of $x$ position

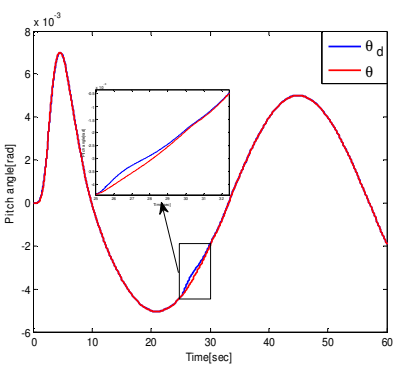

(b) evolution of pitch angle

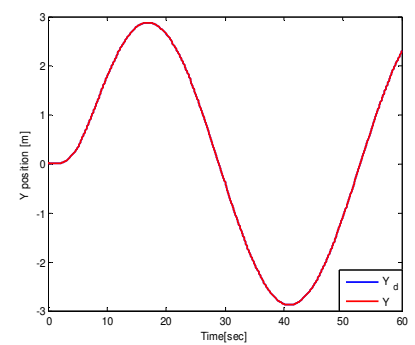

(e) evolution of $y$ position

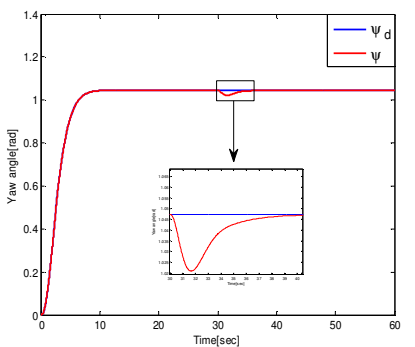

(c) evolution of yaw angle

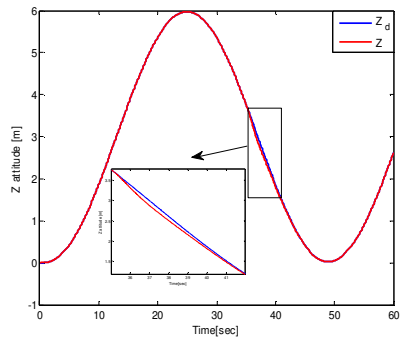

(f) evolution of $z$ position

Figure. 8. Tracking simulation results of trajectories along roll $(\phi)$, pitch $(\theta)$, yaw angle $(\psi)$ and $(\mathrm{X}, \mathrm{Y}, \mathrm{Z})$ axis, Test 2.

Figure. 6 and Figure. 9 represents the inputs control $\left\{u_{1}, u_{2}, u_{3}, u_{4}\right\}$ of our system. From Figure. 9 , it is clear to see the transient peaks in all controllers, especially in inputs control $u_{2}$ and $u_{3}$ which due after each appearance of velocity sensor failures in angular velocities and linear 
velocity of altitude (illustrated respectively by (a), (b), (c), and (f) from Figure. 7) at 20sec, $25 \mathrm{sec}, 30 \mathrm{sec}$ and $35 \mathrm{sec}$. Despite that, the stability of the closed loop dynamics of quadrotor is guaranteed.

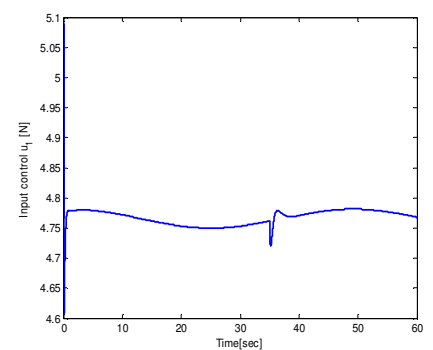

(a) evolution of input control $u_{1}$

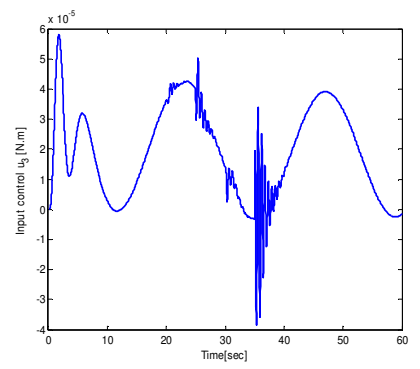

(c) evolution of input control $u_{3}$

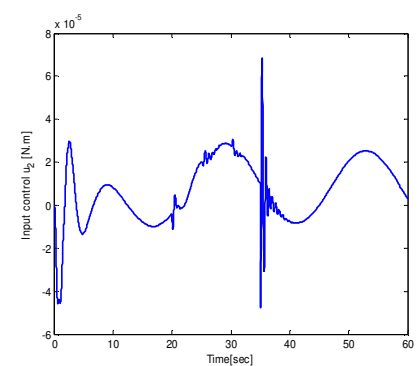

(b) evolution of input control $u_{2}$

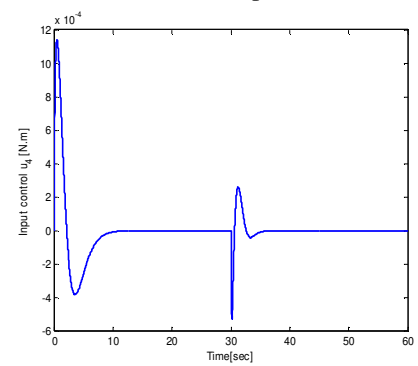

(d) evolution of input control $u_{4}$

Figure. 9. Simulation results of all controllers, Test 2.

We can see also that the obtained input control signals given by the control strategy proposed in this paper are acceptable and physically realizable.

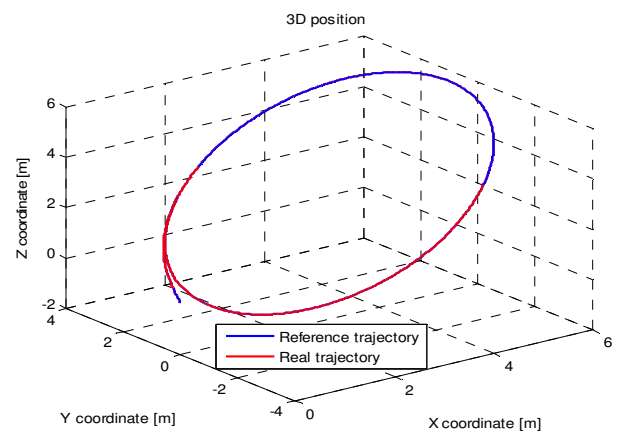

(a) evolution of position along $(X, Y, Z)$ axis, Test 1

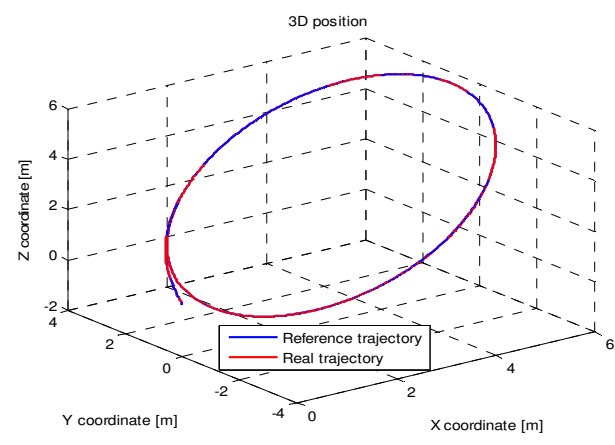

(b) evolution of position along $(X, Y, Z)$ axis, Test 2

Figure. 10. Global trajectory of the quadrotor in 3D.

Figure. 10. represents the 3D position of quadrotor aircraft during the flight. The simulation results given by this figure shows a good performances and robustness towards stability and tracking even after occurrence of velocity sensor failures, which explains the efficiency of control strategy developed in this paper.

\section{CONCLUSIONS AND FUTUR WORKS}

In this paper, we proposed a new control strategy based on robust integral backstepping using sliding mode taking into account the sensor failures. Firstly, we present the dynamical model of 
the quadrotor taking into account the different physics phenomena which can influence the evolution of our system in the space, and secondly by the synthesis a stabilizing control laws in the presence of sensor failures. The simulation results have shown high efficiency of this control strategy, she preserves stability and performances of quadrotor during a malfunction of these velocity sensors. As prospects we hope to implement this control strategy on a real system.

\section{APPENDIX}

Table 1. Quadrotor parameters.

\begin{tabular}{c|l}
\hline Parameter & Value \\
\hline$m$ & $0.486 \mathrm{~kg}$ \\
$g$ & $9.806 \mathrm{~m} / \mathrm{s}^{2}$ \\
$l$ & $0.25 \mathrm{~m}$ \\
$b$ & $2.9842 \times 10^{-5} \mathrm{~N} / \mathrm{rad} / \mathrm{s}$ \\
$d$ & $3.2320 \times 10^{-7} \mathrm{~N} . \mathrm{m} / \mathrm{rad} / \mathrm{s}$ \\
$J_{r}$ & $2.8385 \times 10^{-5} \mathrm{~kg} . \mathrm{m}^{2}$ \\
$I_{(x, y, z)}$ & diag $(3.8278,3.8278,7.1345) \times 10^{-3} \mathrm{~kg} . \mathrm{m}^{2}$ \\
$K_{f a(x, y, z)}$ & diag $(5.5670,5.5670,6.3540) \times 10^{-4} \mathrm{~N} / \mathrm{rad} / \mathrm{s}$ \\
$K_{f(x(x, y) z}$ & diag $(0.0320,0.0320,0.0480) \mathrm{N} / \mathrm{m} / \mathrm{s}$ \\
$k_{m}$ & $4.3 \times 10^{-3} \mathrm{N.m} / \mathrm{A}$ \\
$k_{g}$ & 5.6 \\
$R_{a}$ & $0.67 \Omega$ \\
\hline
\end{tabular}

\section{REFERENCES}

[1] P. Adigbli, C. Grand, J. B. Mouret, \& S. Doncieux. (2007) "Nonlinear attitude and position control of a micro quadrotor using sliding mode and backstepping techniques", Conference and Flight Competition (EMAV), France, pp. 1-9.

[2] S. Bouabdellah, \& R.Siegwart. (2005) "Backstepping and sliding mode techniques applied to an indoor micro quadrotor", Proceeding of the IEEE, ICRA, Barcelona, Spain, pp. 2259-2264.

[3] H. Bouadi, M. Bouchoucha, \& M. Tadjine. (2007) "Modelling and Stabilizing Control Laws Design Based on Backstepping for an UAV Type-Quadrotor", Proceeding of 6 the IFAC Symposium on IAV, Toulouse, France.

[4] H. Bouadi, M. Bouchoucha, \& M. Tadjine. (2007) "Sliding Mode Control Based on Backstepping Approach for an UAV Type-Quadrotor", International Journal of Applied Mathematics and Computer Sciences, Vol. 4, No. 1, pp. 12-17.

[5] H .Bouadi, S. Simoes Cunha, A. Drouin, \& F. Mora-Camino. (2011) "Adaptive Sliding Mode Control for Quadrotor Attitude Stabilization and Altitude Tracking" Proceeding of 12th IEEE International Symposium on Computational Intelligence and Informatics, Budapest, Hungary, pp. 449-455.

[6] M. Bouchoucha, M. Tadjine, A. Tayebi, \& P. Müllhaupt. (2008) "Step by Step Robust Nonlinear PI for Attitude Stabilization of a Four-Rotor Mini-Aircraft", Proceeding of 16th Mediterranean Conference on Control and Automation Congress Centre, Ajaccio, France, pp. 1276-1283.

[7] M. Bouchoucha, S. Seghour, \& M. Tadjine. (2011) "Classical and Second Order Sliding Mode Control Solution to an Attitude Stabilization of a Four Rotors Helicopter: from Theory to Experiment", Proceeding of the IEEE Conference on Mechatronics, Istanboul, Turkey, pp. 162169. 
International Journal of Control Theory and Computer Modelling (IJCTCM) Vol.2, No.2, March 2012

[8] P. Castillo, A. Dzul, \& R. Lozano. (2004) "Real-Time Stabilization and Tracking of a Four-Rotor Mini Rotorcraft", IEEE Transactions on Control Systems Technology, Vol. 12, No. 4, pp. 510516.

[9] J. Colorado, A. Barrientos, , A. Martinez, B. Lafaverges, \& J. Valente. (2010) "Mini-quadrotor attitude control based on Hybrid Backstepping \& Frenet-Serret Theory", Proceeding of the IEEE, ICRA, Anchorage, Alaska, USA, pp. 1617-1622.

[10] A. Das, F. Lewis, K. Subbarao (2009) "Backstepping Approach for Controlling a Quadrotor Using Lagrange Form Dynamics.” Journal of Intelligent and Robotic Systems, Vol. 56, No. 1-2, pp. 127-151.

[11] L. Derafa, T. Madani, \& A. Benallegue. (2006) "Dynamic modelling and experimental identification of four rotor helicopter parameters”, IEEE-ICIT , Mumbai, India, pp. 1834-1839.

[12] N. Guenard, T. Hamel, \& R. Mahony. (2008) "A Practical Visual Servo Control for an Unmanned Aerial Vehicle”, IEEE Transactions on Robotics, Vol. 24, No. 2, pp. 331-340.

[13] M. Huang, B. Xian, C. Diao, K. Yang, \& Y. Feng. (2010) "Adaptive tracking control of underactuated quadrotor unmanned aerial vehicles via backstepping", Proceeding of American Control Conference, Baltimore, USA, pp. 2076-2081.

[14] R. Lozano, P. Castillo, \& A. Dzul. (2004) "Global stabilization of the PVTOL: real time application to a mini aircraft", International Journal of Control, Vol 77, No 8, pp. 735-740.

[15] T. Madani \& A. Benallegue. (2006) "Backstepping control for a quadrotor helicopter", Proceeding of the IEEE Conference on Intelligent Robots and Systems, pp. 3255-3260.

[16] T. Madani \& A. Benallegue. (2006) "Backstepping Sliding Mode Control Applied to a Miniature Quadrotor Flying Robot", Proceeding of the IEEE Conference on Industrial Electronics, pp. 700705.

[17] C. Nicol, C. J. B. Macnab, \& A. Ramirez-Serrano. (2008) "Robust neural network control of a quadrotor helicopter" Proceeding of the IEEE Canadian Conference on Electrical and Computer Engineering, pp. 1233-1237.

[18] S. Seghour, M. Bouchoucha, \& H. Osmani. (2011) "From Integral Backstepping to Integral Sliding Mode Attitude Stabilization of a Quadrotor System: Real Time Implementation on an Embedded Control System Based on a dsPIC $\mu \mathrm{C}$ ", Proceeding of the IEEE Conference on Mechatronics, Istanboul, Turkey, pp. 154-161.

[19] J.J.E. Slotine, \& W. Li. (1991) “Applied nonlinear control”, Prentice Hall, Englewood Cliffs, NJ.

[20] A. Tayebi, \& S. McGilvray. (2006) "Attitude stabilization of a VTOL Quadrotor Aircaft", IEEE Transactions on Control Systems Technology, Vol. 14, No. 3, pp. 562-571. 
International Journal of Control Theory and Computer Modelling (IJCTCM) Vol.2, No.2, March 2012

Authors

Mr. Hicham KHEBBACHE is Graduate student (Magister) of Automatic Control at the Electrical Engineering Department of Setif University, ALGERIA. He received the Engineer degree in Automatic Control from Jijel University, ALGERIA in 2009. He is with Automatic Laboratory of Setif (LAS). His research interests include Aerial robotics, Linear and Nonlinear control, Robust control, Fault tolerant control (FTC), Diagnosis, Fault detection and isolation (FDI).

Mr. Belkacem SAIT is Associate Professor at Setif University and member at Automatic Laboratory of Setif (LAS), ALGERIA. He received the Engineer degree in Electrical Engineering from National Polytechnic school of Algiers (ENP) in 1987, Magister degree in Instrumentation and Control from HCR of Algiers in 1992, and Ph.D. in Automatic Product from Setif University in 2007. His research interested include discrete event systems, hybrid systems, Petri nets, Fault tolerant control (FTC), Diagnosis, Fault detection and isolation (FDI).

Mr. Fouad YACEF is currently a Ph.D. student at the Automatic Control Department of Jijel University, ALGERIA. He received the Engineer degree in Automatic Control from Jijel University, ALGERIA in 2009, and Magister degree in Control and Command from Military Polytechnic School (EMP), Algiers, in November 2011. He is with Automatic Laboratory of Jijel (LAJ). His research interests include Aerial robotics, Linear and Nonlinear control, LMI optimisation, Analysis and design of intelligent control systems.
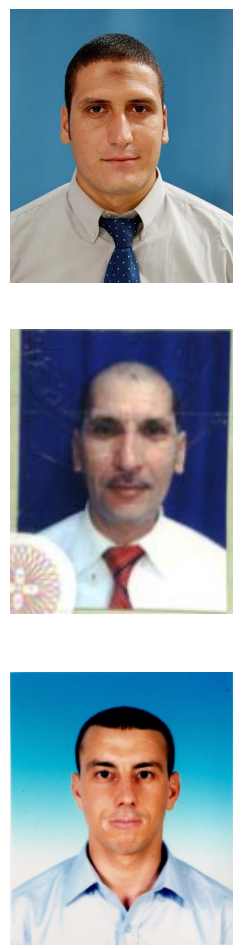